\title{
Leukocyte RhoA exchange factor Arhgef1 mediates vascular inflammation and atherosclerosis
}

\author{
Maria Luigia Carbone, ${ }^{1}$ Gilliane Chadeuf,, Sandrine Heurtebise-Chrétien, ${ }^{1}$ Xavier Prieur, ${ }^{1}$ Thibault Quillard, ${ }^{2}$ Yann Goueffic, ${ }^{2,3}$ \\ Nathalie Vaillant, ${ }^{1}$ Marc Rio, ${ }^{1}$ Laure Castan, ${ }^{1}$ Maxim Durand, ${ }^{4}$ Céline Baron-Menguy, ${ }^{1}$ Julien Aureille, ${ }^{1}$ Juliette Desfrançois, ${ }^{5}$ \\ Angela Tesse, ${ }^{1}$ Raul M. Torres, ${ }^{6}$ and Gervaise Loirand ${ }^{1,3}$ \\ ${ }^{1} I N S E R M$, CNRS, UNIV Nantes, l'institut du thorax, Nantes, France. INSERM, UNIV Nantes, Laboratoire de Physiopathologie de la Résorption Osseuse et thérapie des tumeurs osseuses primitives, Nantes, \\ France. ${ }^{3} \mathrm{CHU}$ de Nantes, Nantes, France. ${ }^{4}$ INSERM, UNIV Nantes, Institut de Transplantation Urologie Néphrologie, France. ${ }^{5}$ Cytocell Platform, Institut de Recherche en Santé-UNIV Nantes, Nantes, France. \\ ${ }^{6}$ Department of Immunology and Microbiology, University of Colorado School of Medicine, Aurora, Colorado, USA.
}

\begin{abstract}
Abnormal activity of the renin-angiotensin-aldosterone system plays a causal role in the development of hypertension, atherosclerosis, and associated cardiovascular events such as myocardial infarction, stroke, and heart failure. As both a vasoconstrictor and a proinflammatory mediator, angiotensin II (Ang II) is considered a potential link between hypertension and atherosclerosis. However, a role for Ang II-induced inflammation in atherosclerosis has not been clearly established, and the molecular mechanisms and intracellular signaling pathways involved are not known. Here, we demonstrated that the RhoA GEF Arhgef1 is essential for Ang II-induced inflammation. Specifically, we showed that deletion of Arhgef1 in a murine model prevents Ang II-induced integrin activation in leukocytes, thereby preventing Ang II-induced recruitment of leukocytes to the endothelium. Mice lacking both LDL receptor (LDLR) and Arhgef1 were protected from high-fat diet-induced atherosclerosis. Moreover, reconstitution of $\mathrm{LdIr}^{-/-}$mice with Arhgef1-deficient BM prevented high-fat diet-induced atherosclerosis, while reconstitution of $\mathrm{LdIr}^{-1-}$ Arhgef1 ${ }^{-/-}$with WT BM exacerbated atherosclerotic lesion formation, supporting Arhgef1 activation in leukocytes as causal in the development of atherosclerosis. Thus, our data highlight the importance of Arhgef1 in cardiovascular disease and suggest targeting Arhgef1 as a potential therapeutic strategy against atherosclerosis.
\end{abstract}

\section{Introduction}

Angiotensin II (Ang II) is considered as the main mediator of both the physiological and the pathophysiological actions of the renin-angiotensin-aldosterone system (RAAS) through the stimulation of the Ang II type 1 (AT1) receptor, a ubiquitously expressed $G$ protein-coupled receptor able to recruit numerous intracellular signaling pathways $(1,2)$. Consequently, AT1 receptor activation in both arterial wall (smooth muscle and endothelial) and circulating (leukocytes and platelets) cells leads to pleiotropic actions affecting arterial contraction, vascular permeability, hemostasis, immune/inflammatory cell activation, and oxidative stress (3). Pharmacological inhibition or genetic disruption of the RAAS decreases atherosclerosis in various experimental models (4-11), and clinical trials support this pro-atherosclerotic and proinflammatory role of Ang II in humans $(12,13)$. However, the respective contribution of leukocytes and arterial wall cells in the effect of Ang II on inflammation and atherosclerosis is still not clearly established, and the intracellular signaling pathways involved remained largely unknown. We explored these mechanisms by focusing on the role of RhoA signaling, the inhibition of which mediates, at least in part, the pleiotropic effects of statins and

Authorship note: G. Chadeuf and S. Heurtebise-Chrétien contributed equally to this work. Conflict of interest: The authors have declared that no conflict of interest exists.

Submitted: January 9, 2017; Accepted: October 5, 2017.

Reference information: J Clin Invest. 2017;127(12):4516-4526.

https://doi.org/10.1172/JCI92702 their beneficial effects on cardiovascular diseases $(14,15)$. We particularly address the role of Arhgef1, the RhoA GEF responsible for AT1 receptor stimulation-induced Jak2-dependent RhoA activation in rodent and human vascular and circulating cells (16, 17). Activation of Arhgef1 by AT1 receptor stimulation in vascular smooth muscle cells is responsible for Ang II-induced vasoconstriction, increased vascular tone, and hypertension (17). We hypothesized that, in addition to its critical role in blood pressure regulation and hypertension, Arhgef1 is involved in the proinflammatory and pro-atherosclerotic effects of Ang II.

\section{Results}

Arhgef1 deletion prevents Ang II-induced leukocyte recruitment. We first analyzed the proinflammatory action of Ang II by measuring Ang II-induced leukocyte-endothelium interaction in vivo in Arhgef1 $1^{-/-}$mice by intravital microscopy. "Arhgef1 $1^{-/}$mice" refers to mice with constitutive knockout of the Arhgef1 gene in Arhgef1 $1^{\text {lox/lox }}$ mice mated to CMV-Cre deleter mice. Ang II induced a time-dependent and losartan-sensitive increase in leukocyte rolling and adhesion in Arhgef ${ }^{\text {lox/lox }}$ mice that was strongly reduced in Arhgef1 ${ }^{-/-}$mice, while blood cell count was similar (Figure 1, A and B, and Supplemental Figures 1 and 2; supplemental material available online with this article; https://doi.org/10.1172/JCI92702DS1). This inhibition of Ang II-induced leukocyte recruitment in Arhgef1 $1^{-/-}$mice was associated with a reduction of circulating proinflammatory cytokines in Arhgef1 $1^{-/-}$mice compared with Arhgef1 $1^{\text {lox/lox }}$ 
A

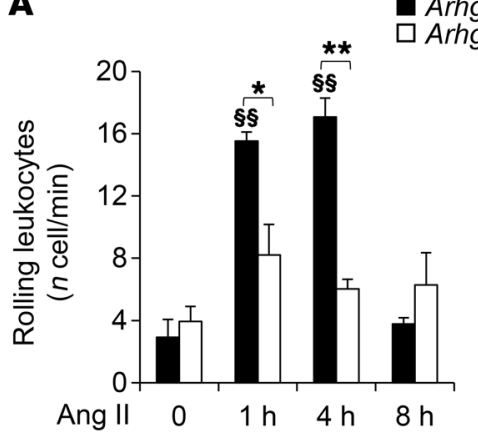

C

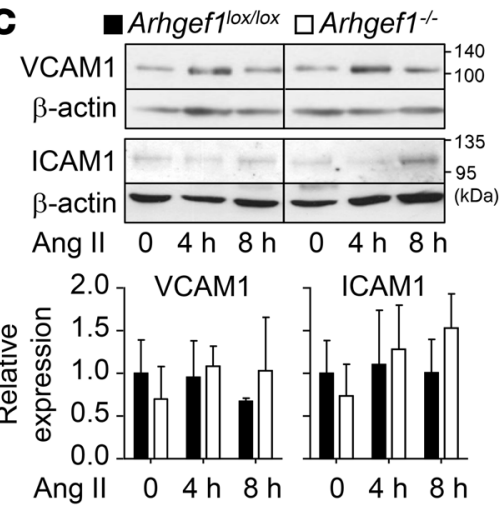

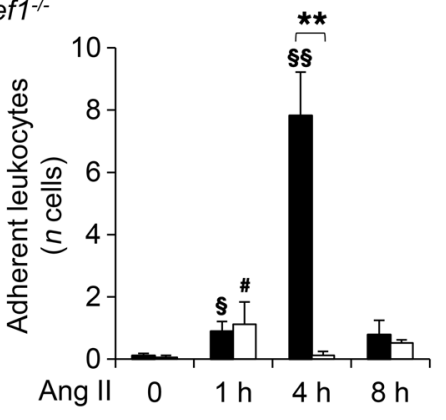

D
B

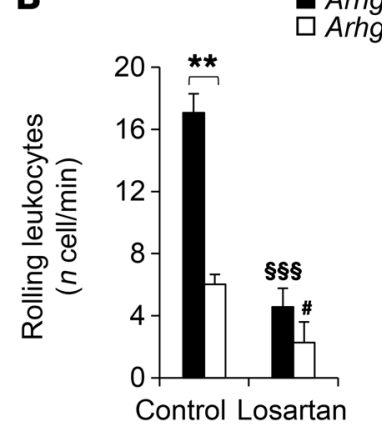

Arhgef1/ox/10x

(10

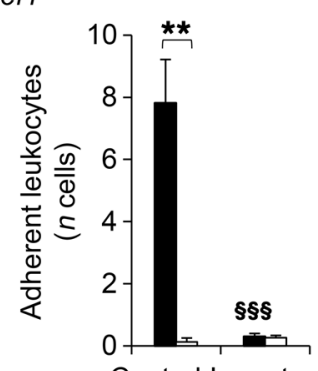

Control Losartan
E
Arhgefflox/lox
Arhgef1 $1 /$ -

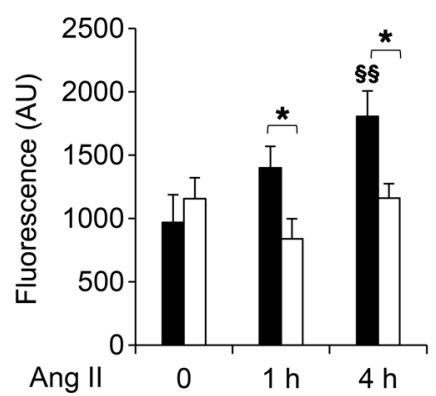

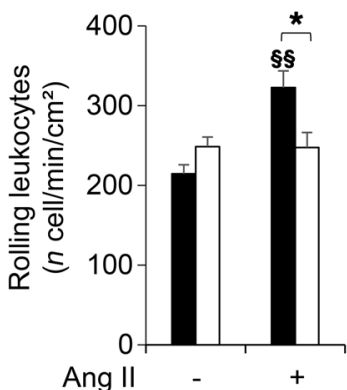

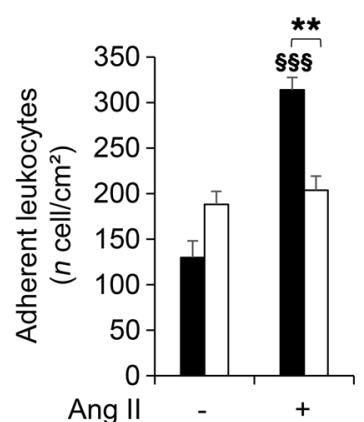

Figure 1. Deletion of Arhgef1 inhibits leukocyte rolling and adhesion. (A) Time-dependent in vivo effect of Ang II (30 pmol) on leukocyte rolling and adhesion in mesenteric vessels of Arhgef1 ${ }^{\text {lox/lox }}$ and Arhgef1/- mice ( $n=5$ mice). (B) Effect of losartan on leukocyte rolling and adhesion induced by Ang II (30 pmol, 4 hours) in mesenteric vessels of Arhgef1 ${ }^{10 x / l o x}$ and Arhgef1 ${ }^{-/-}$mice ( $n=5$ mice). (C) Representative immunoblot of VCAM1, ICAM1, and $\beta$-actin in lysates of aortas from Arhgef1/ox/lox and Arhgef1-/- mice before (0) and 4 and 8 hours after Ang II treatment ( $n=3$ ) and corresponding quantification. All lanes were run on the same gel, but lanes 3 and 4 were noncontiguous as indicated by the black dividing line. (D) In vitro static adhesion of Arhgef1lox/lox

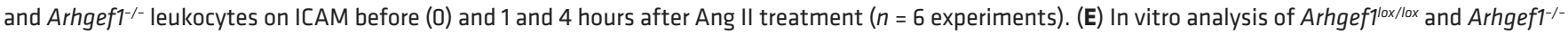
leukocyte rolling and adhesion on HUVECs under shear flow, before (-) and 4 hours after $(+)$ Ang II treatment $(n=5)$. ${ }^{*} P<0.05$, ${ }^{* *} P<0.01$, Arhgef1/ox/lox vs.

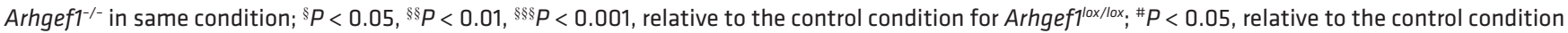
for Arhgef1 ${ }^{-/-}$; Mann-Whitney.

mice (Supplemental Figure 3). To discriminate between the roles of endothelial cells and leukocytes in the decreased Ang II-induced leukocyte rolling and adhesion caused by Arhgef1 deletion, we next analyzed the endothelial expression of vascular cell adhesion molecule-1 (VCAM1) and intercellular adhesion molecule-1 (ICAM1) (Figure 1C). Both in basal condition and after Ang II stimulation, the expression of VCAM1 and ICAM1 was similar in Arhgef1 $1^{-/-}$and Arhgef1 $1^{\text {lox } / \text { lox }}$ mice, suggesting that the reduced recruitment of leukocytes resulted not from a downregulation of endothelial adhesion molecules but rather from an alteration of leukocyte binding. To confirm this hypothesis, we compared the ability of Arhgef1 $1^{-/-}$and Arhgef1 ${ }^{\text {lox/lox }}$ leukocytes to adhere in vitro on ICAM1 under static conditions and on HUVEC monolayers under flow conditions (Figure 1, D and E). Basally, adhesion of Arhgef1 $1^{-/-}$and Arhgef1 $1^{\text {lox/lox }}$ leukocytes to ICAM1 was similar. However, Ang II stimulation increased the adhesion of Arhgef1 ${ }^{\text {lox/lox }}$ leukocytes on ICAM1 but had no effect on Arhgef1 $1^{-/-}$leukocytes (Figure 1D). Similarly, in the in vitro flow chamber assay on HUVEC monolayers, Arhgef1 deletion prevented Ang II-induced stimulation of leukocyte rolling and adhesion on HUVECs (Figure 1E). These in vitro results thus support an essential role of leukocytes in the impairment of leukocyte-endothelium interaction in Arhgef1 $1^{-/-}$mice.
Arhgef1 deletion in leukocytes prevents Ang II-induced leukocyte recruitment. To prove that the loss of Arhgef1 expression in leukocytes is indeed the primary cause of the altered Ang II-induced leukocyte adhesion to the endothelium in vivo, we generated several combinations of bone marrow (BM) chimeric mice. We confirmed successful transplantation (Supplemental Figure 4) and analyzed leukocyte-endothelium interaction by intravital microscopy (Figure 2A). As expected, Arhgef1 ${ }^{\text {lox/lox }}$ Arhgef1 ${ }^{\text {lox/lox }}$ and Arhgef1 $1^{-/}$Arhgef1 $1^{-/-}$ chimeric mice reproduced the phenotype of Arhgef1 ${ }^{\text {lox/lox }}$ and Arhgef1 $1^{-/-}$mice, respectively, with a marked stimulation of leukocyte rolling and adhesion by Ang II in Arhgef1 $1^{\text {lox/lox}}$ Arhgef $1^{\text {lox/lox }}$ mice but not in Arhgef1 $1^{-/}$Arhgef1 $1^{-/}$mice (Figure 2A). In Arhgef1 $1^{-/-}$Arhgef1 ${ }^{\text {lox/lox }}$ chimeric mice that lacked Arhgef1 only in hematopoietic cells, the stimulatory effect of Ang II on leukocyte adhesion and rolling was lost (Figure 2A). In contrast, repopulation of Arhgef1 ${ }^{-/-}$recipient with Arhgef $1^{\text {lox/lox }} \mathrm{BM}$ restored leukocyte rolling and adhesion response to Ang II (Figure 2A). These chimeric models thus demonstrate that the defective Ang II-induced leukocyte rolling and adhesion in Arhgef1 $1^{-/-}$mice were due to the loss of Arhgef1 expression in leukocytes.

Arhgef1 deletion in leukocytes affects $\beta_{2}$ integrin activation. Adhesion of leukocytes to endothelial cells and ICAM1 is mediated by the $\beta_{2}$ integrins, mainly LFA-1 (CD18/CD11a) and Mac-1 (CD18/ $\mathrm{CD} 11 \mathrm{~b})$. To assess a potential role of Arhgef1 in the regulation of 
A
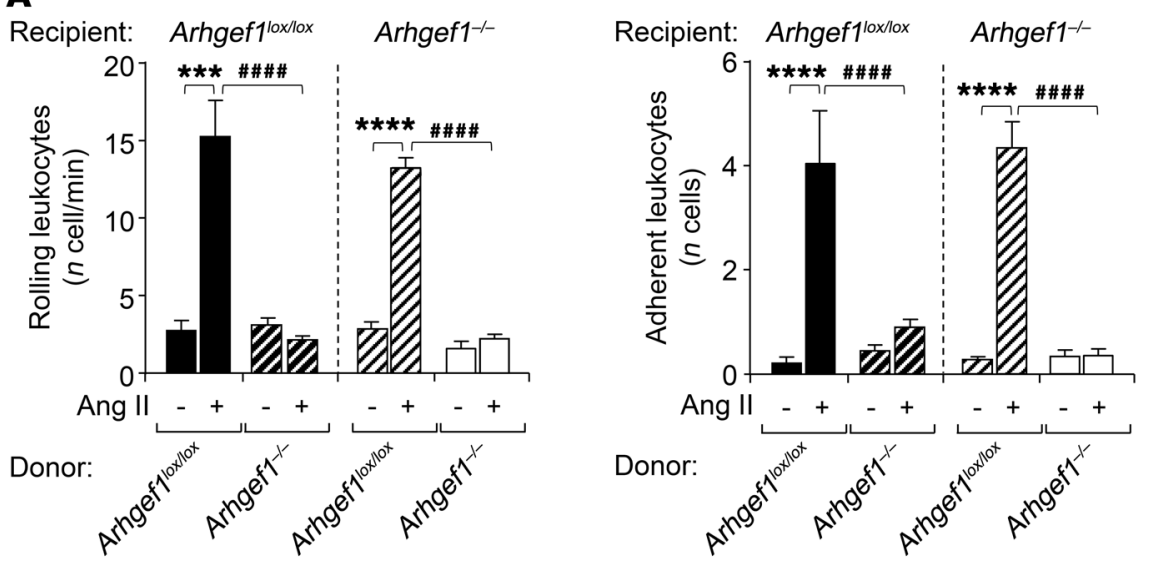

B

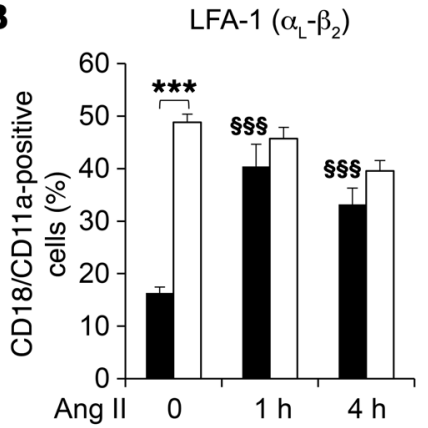

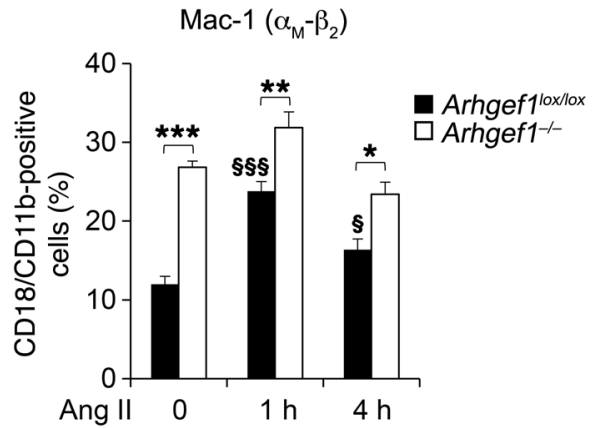

C

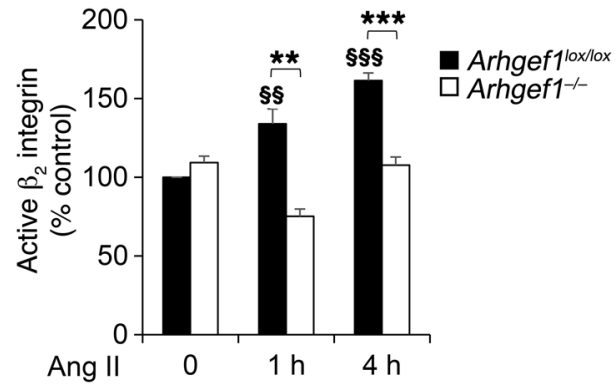

Figure 2. Deletion of the RhoA exchange factor Arhgef1 in leukocytes inhibits Ang II-induced leukocyte rolling and adhesion, and $\beta_{2}$ integrin activation. (A) In vivo leukocyte rolling and adhesion in chimeric mice before $(-)$ and 4 hours after Ang II treatment $\left(30\right.$ pmol) $(n=5)$. ${ }^{* * *} P<0.001$, ${ }^{* * * *} P<0.0001$,

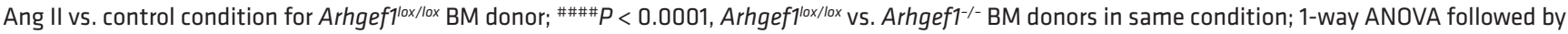
Sidak post hoc test. (B) Expression of LFA-1 and Mac-1 in leukocytes from Arhgeff $1^{\text {lox } / \text { lox }}$ and Arhgef1 $1^{-1-}$ mice before (0) and 1 and 4 hours after Ang II treatment $(n=5)$. (C) Expression of the active, high-affinity $\beta_{2}$ integrin in leukocytes from Arhgef1ox/lox and Arhgef1 $1^{-/-}$mice before ( 0 ) and 1 and 4 hours after Ang II treatment $(n=4) .{ }^{*} P<0.05,{ }^{* *} P<0.01,{ }^{* * *} P<0.001$, Arhgef10x/lox vs. Arhgef1-1/ in same condition; $\$ P<0.05,{ }^{\$ \S} P<0.01,{ }^{\S \S \S} P<0.001$, relative to the control (0) condition for Arhgef1 ${ }^{10 x / 10 x}$; Mann-Whitney in $\mathbf{B}$ and $\mathbf{C}$.

the expression and/or the conformational state of the $\beta_{2}$ integrins, we first determined the membrane expression of LFA-1 and Mac-1 in Arhgef1 $1^{\text {lox/lox }}$ and Arhgef1 $1^{-/-}$leukocytes. In Arhgef1 $1^{\text {lox/lox }}$ leukocytes, Ang II stimulation induced an increase in both LFA-1 and Mac1 expression (Figure 2B). Surprisingly, under basal conditions, LFA-1 and Mac-1 expression was upregulated in Arhgef1 $1^{-1-}$ leukocytes compared with Arhgef1 $1^{l o x} / l o x$, but was not increased by Ang II (Figure 2B). $\beta_{2}$ Integrin activation results in a conformational change that depends on both RhoA- and Rap-dependent signaling (18-20). This conversion to a high-affinity open conformation leads to the formation of the epitope for the 24 antibody (mAb 24). We therefore used this activation reporter epitope to assess the expression of high-affinity $\beta_{2}$ integrins at the membrane of Arhgef1 $1^{\text {lox/lox }}$ and Arhgef1 $1^{-/-}$leukocytes (Figure 2C). Under resting condition, the expression of high-affinity $\beta_{2}$ integrins is similar in Arhgef $1^{\text {lox/lox }}$ and Arhgef1 $1^{-/-}$leukocytes (Figure 2C). Ang II increased the expression of high-affinity $\beta_{2}$ integrins in Arhgef $1^{\text {lox } / \text { lox }}$ but not in Arhgef1 $1^{-/-}$leukocytes, indicating the requirement of Arhgef1 for the regulation of the $\beta_{2}$ integrin conformational state in leukocytes.

Arhgef1 deletion prevents atherosclerosis. Because recruitment of leukocytes, involving both leukocyte $\beta_{2}$ integrins (21) and AT1 receptors (7), is critical in the development of atherosclerotic lesions, we hypothesized that Arhgef1 participates in the pathogenesis of atherosclerosis. We therefore analyzed the development of high-fat diet-induced atherosclerosis in $\mathrm{Ldlr}^{-1}$ Arhgef1 ${ }^{-/-}$ double-knockout mice compared with $\mathrm{Ldll}^{-1}$ Arhgef fox/lox mice (Figure 3). Both en face Oil Red O staining of the complete aorta and analysis of aortic root sections revealed a significant reduction in atherosclerotic lesion formation in $\mathrm{Ldlr}^{-/}$Arhgef1 ${ }^{-/-}$mice (Figure 3A), despite a similar systolic arterial pressure and plasma total cholesterol level in the 2 groups of mice (Figure 3, B and C). Moreover, Arhgef1 genotype did not affect the plasma lipid profile, and the uptake of oxidized LDL by peritoneal macrophages isolated from the 2 groups of mice was the same (Figure 3, D and E). In agreement with these functional observations, the expression of the macrophage scavenger receptor CD36 and the major lipid droplet protein ADRP was not modified by Arhgef1 deletion (Supplemental Figure 5), indicating that the reduction of atherosclerotic lesions in $\mathrm{Ldlr}^{-/}$Arhgef1 ${ }^{-/-}$mice was not related to a reduced macrophage lipid uptake. These results suggest that neither a modification of lipid metabolism nor a change in hemodynamic condition accounts for the reduced atherosclerosis in $\mathrm{Ldlr}^{-/}$ Arhgef1 $1^{-/}$mice compared with Ldlr/-Arhgef1 ${ }^{\text {lox } / l o x}$ mice. Immunofluorescence analysis of aortic root sections using anti-CD3 and 
A

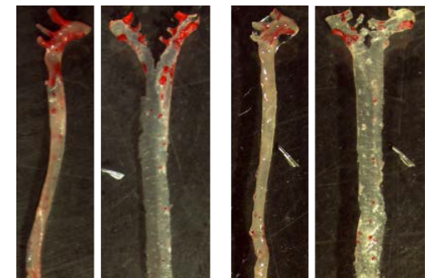

Ldlir-Arhgef 10x/lox LdIr $^{-1-}$ Arhgef 1-1-

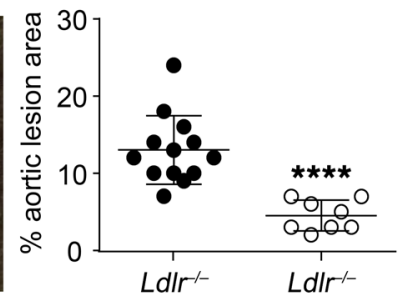

Arhgef 110x/lox Arhgef $1^{-/}$

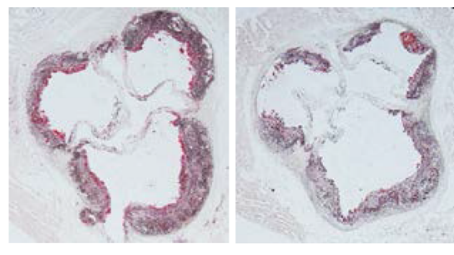

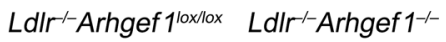

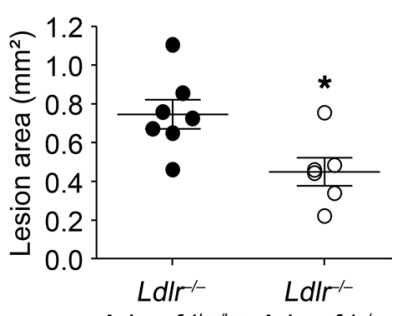

Arhgef 1 $1^{10 x / 10 x}$ Arhgef $1^{-1-}$

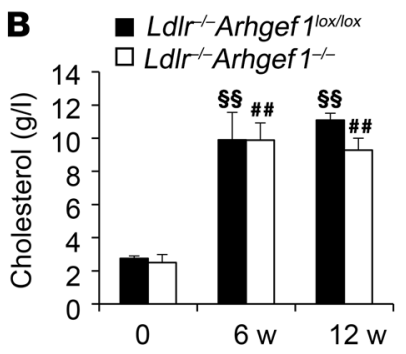

$\mathbf{E}$

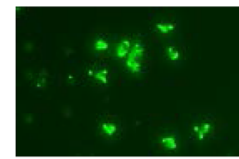

Ldll'r-Arhgef $1^{10 \times / 10 x}$

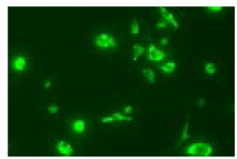

Ldlr ${ }^{-1}$ Arhgef $1^{-1-}$

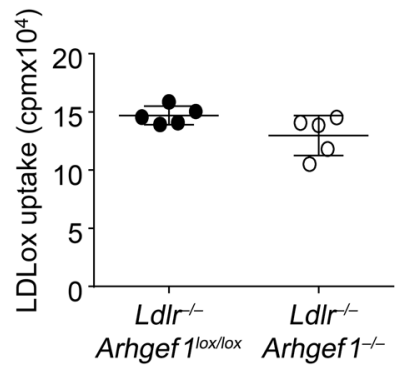

C

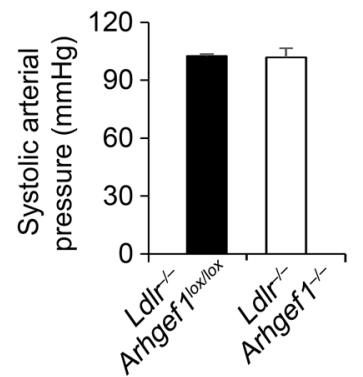

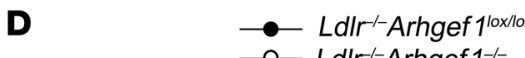

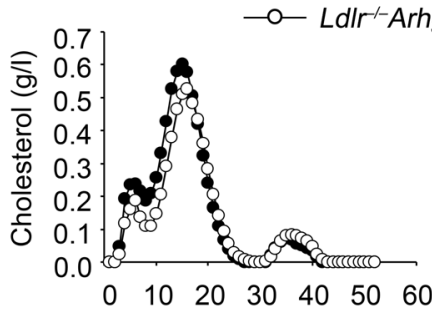

$\mathbf{F}$
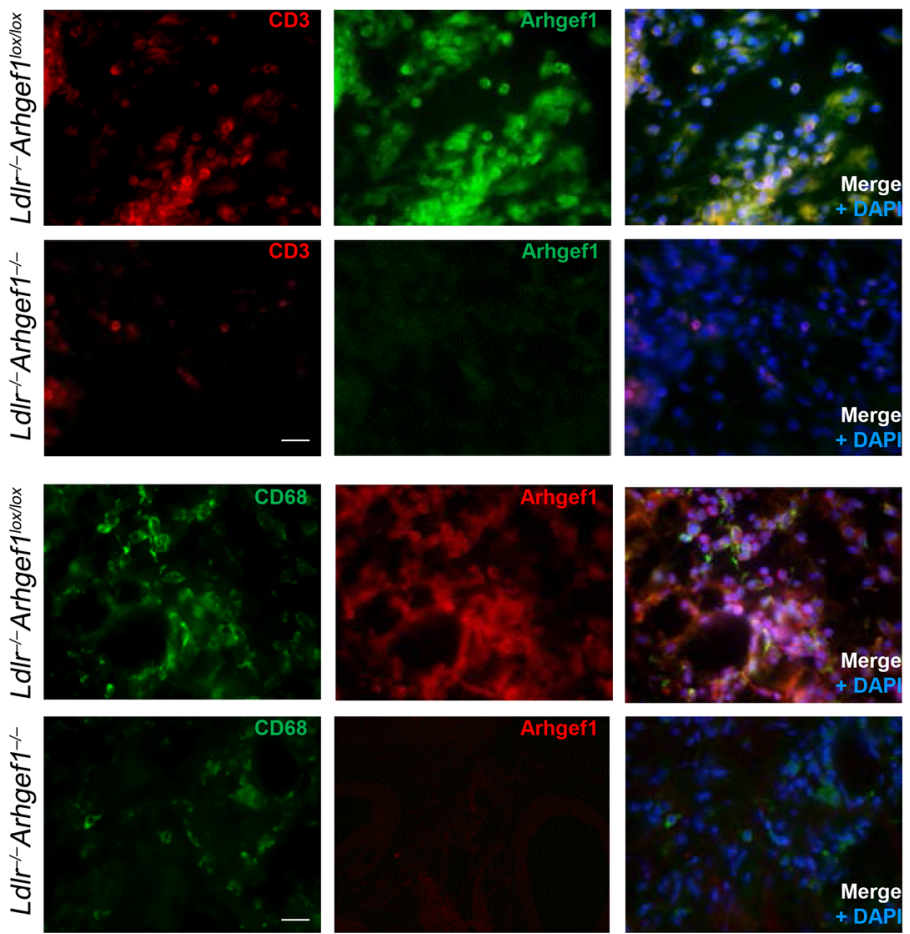

Figure 3. Deletion of the RhoA exchange factor Arhgef1 inhibits atherosclerosis. (A) Quantification of atherosclerotic lesions in whole aorta and the aortic

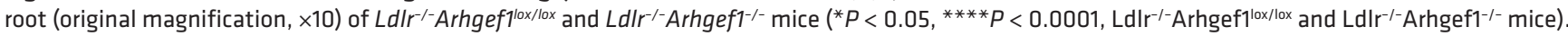

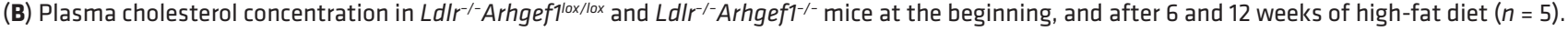
$\$ \$ P<0.01$, relative to the control (0) condition for Arhgef1/0x/lox; \#\#P<0.01, relative to the control (0) condition for Arhgef1 $1^{-1-}$. (C) Systolic blood pressure of

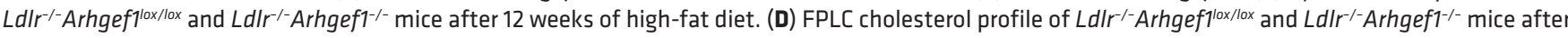

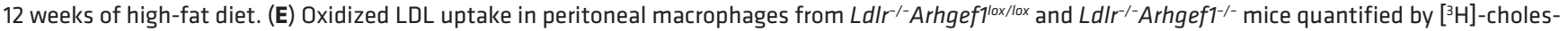
terol uptake and illustrated above by BODIPY staining. (F) Representative images of CD3, CD68, and Arhgef1 staining of aortic root sections from Ldlr ${ }^{-1-}$ Arhgef10x/lox and Ldlr ${ }^{-1-}$ Arhgef1 $1^{-1-}$ mice $(n=6$; scale bars: $20 \mu \mathrm{m})$. Mann-Whitney test in $\mathbf{A}-\mathbf{C}$ and $\mathbf{E}$; 1 -way ANOVA followed by Bonferroni post hoc test in $\mathbf{D}$.

anti-CD68 antibodies revealed that Arhgef1 deficiency reduced the infiltration of lymphocytes and monocytes into the lesions (Figure $3 \mathrm{~F}$ and Figure 4A). This observation was confirmed by flow cytometry analysis that showed a significant reduction of the number of leukocytes, $\mathrm{T}$ lymphocytes, and macrophages in atherosclerotic aortas from $L d l r^{-/}$Arhgef1 $1^{-/}$mice compared with $L d l r^{-/}$Arhgef1 ${ }^{l o x / l o x}$ mice (Figure 4B). In agreement with these observations, the level of the macrophage marker F4/80 transcript (Adgre1) was found to be significantly lower in atherosclerotic aortas from $\mathrm{Ldlr}^{-1} \mathrm{Arh}$ -

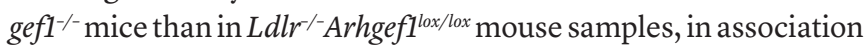


A

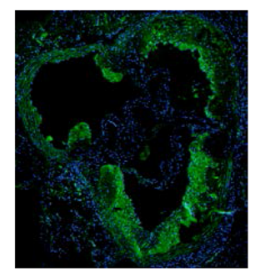

Ldlr-'-

Arhgef1/0x/lox

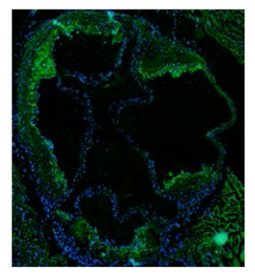

LdIr $r^{-}$

Arhgef1-r

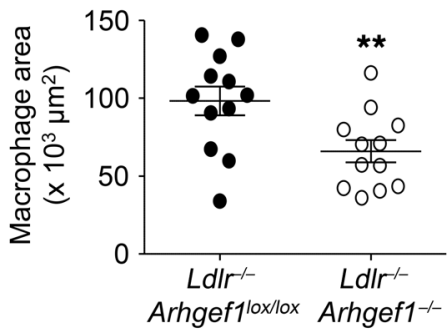

B

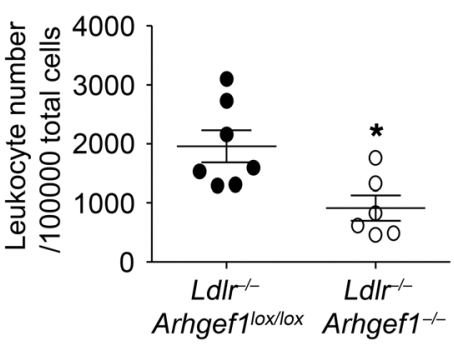

C

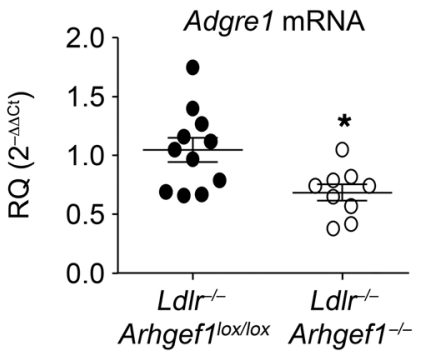

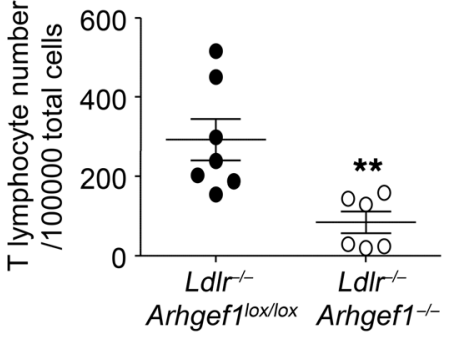

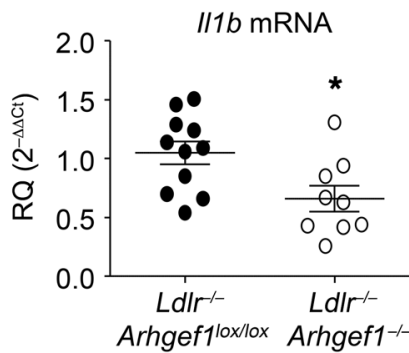

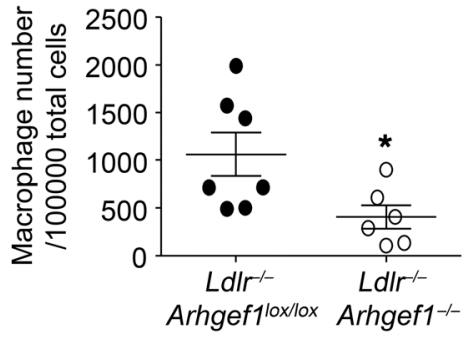

Tnfa mRNA

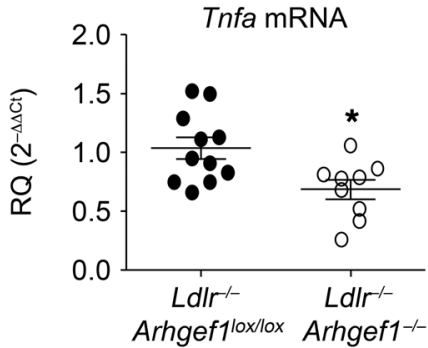

Figure 4. Deletion of Arhgef1 decreases immune cell accumulation and inflammation in atherosclerotic aorta. (A) Representative photomicrographs of CD68

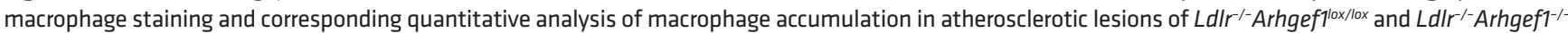
mice (original magnification, $\times 10$; ${ }^{*} P<0.05$, Mann-Whitney test). (B) Quantification of total leukocytes, T lymphocytes, and macrophages in atherosclerotic

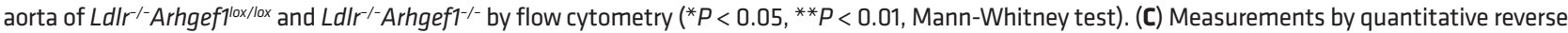
transcriptase PCR of mRNA levels of the macrophage markers Adgre1, II1b, and Tnfa (encoding F4/80, IL-1 1 , and TNF- $\alpha$, respectively) in atherosclerotic aorta of

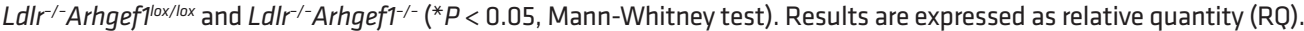

with a decreased level of Illb and Tnfa transcripts (Figure 4C). In contrast, Icam 1 and Vcam 1 transcript levels were similar in athero-

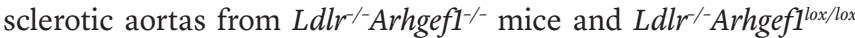
mice, indicating that endothelial cell activation/dysfunction was not affected by Arhgef1 deletion (Supplemental Figure 6). In vitro experiments showed that Arhgefi deficiency did not affect cytokine secretion by isolated T cells (Supplemental Figure 7). All together these results strongly suggest that the reduction of atherosclerosis and lesion inflammation in $\mathrm{Ldlr}^{-1}$ Arhgef ${ }^{-/}$mice resulted from the decreased number of the immune cells in atherosclerotic lesions. This reduction of immune cell accumulation in plaques induced by Arhgefl deletion is in agreement with the role of Arhgef1 in mediating adhesion of leukocytes to the endothelium and suggests that the protective role of Arhgef1 deletion against atherosclerosis in the $\mathrm{Ldlr} /$ mouse model was mainly due to the loss of Arhgef1 expression in leukocytes.

Arhgef1 deletion in leukocytes prevents atherosclerosis. To confirm the contribution of leukocyte Arhgef1 to atherogenesis, both

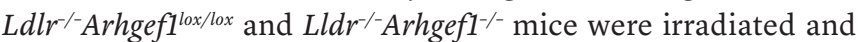
repopulated with BM cells from $L d l r^{\prime}-A r h g e f 1^{10 x / l o x}$ and $L d l r^{\prime} / A r-$ heef1 $1^{-/}$mice. The size of atherosclerotic lesions was significant- ly reduced in $\mathrm{Ldlr}^{-1}$ Arhgef $1^{10 x / l o x}$ mice with transplanted $\mathrm{Ldlr}^{-1}$ Arhgef1 $1^{-/}$BM that expressed Arhgef1 in all tissues excepted in hematopoietic cells (Figure 5A) despite similar plasma lipid profiles (Figure 5, B and C). By contrast, atherosclerotic lesions were enhanced in Ldlr--Arhgef1 ${ }^{--}$mice reconstituted with Ldlr/-Arhgef1 $1^{10 x / l o x}$ that only expressed Arhgef1 in hematopoietic cells (Figure 5A). This result shows that Arhgef1 genotype of leukocytes confers the atherosclerosis phenotype to $\mathrm{Ldlr}^{-1}$ mice, thus indicating an essential role of leukocyte Arhgef1 in the formation of atherosclerotic lesions in mice.

Arhgef1 is expressed in inflammatory cells in human atherosclerotic lesions. In humans, activation of the RAAS leads to Jak2-mediated activation of Arhgef1 in leukocytes (16). In order to assess whether leukocyte Arhgef1 may play a role similar to that observed in mice in the pathogenesis of atherosclerosis in humans, we performed Arhgef1 immunostaining in human carotid atherosclerotic lesions (Figure 6). Immunohistochemical staining shows that Arhgef1 is expressed in vascular endothelial and smooth muscle cells but the strongest expression level is observed in immune cells, with a correlation between Arhgef1 and CD3-positive areas in atherosclerotic lesions (Figure 6A). 
A
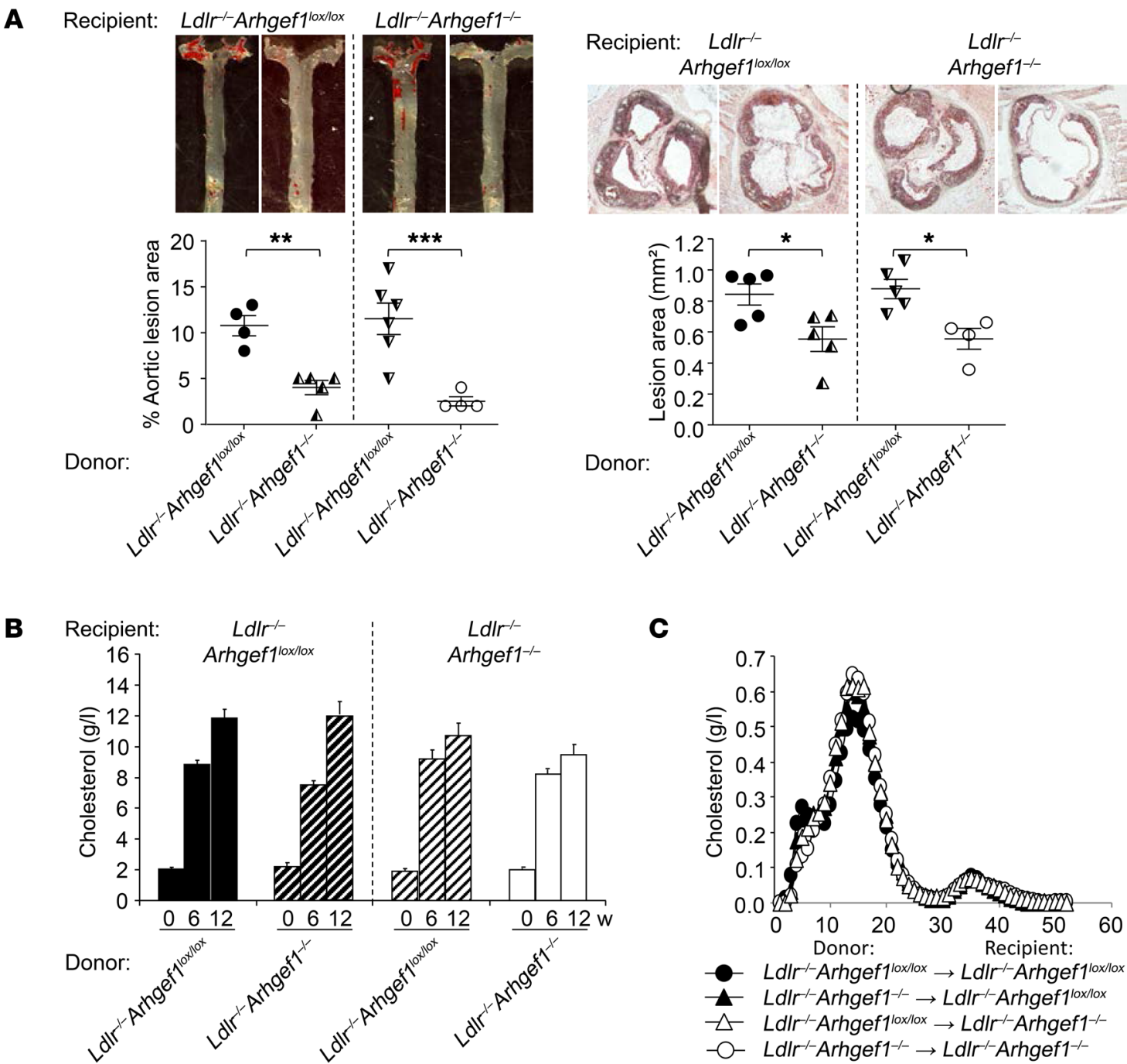

Figure 5. Deletion of the RhoA exchange factor Arhgef1 in leukocytes inhibits atherosclerosis. (A) Quantification of atherosclerotic lesions in whole aorta and

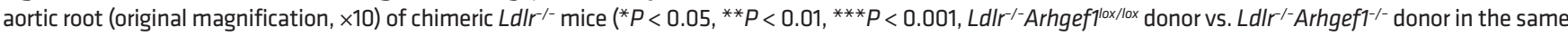

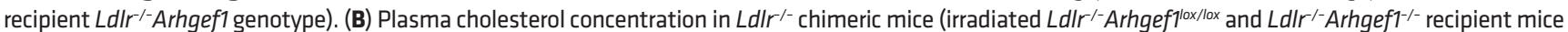

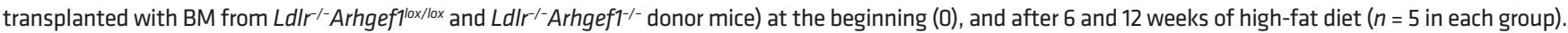
(C) FPLC cholesterol profile in Ldlr/- chimeric mice after 12 weeks of high-fat diet ( $n=5$ in each group). 1-way ANOVA followed by Bonferroni post hoc test.

Coimmunofluorescent staining shows that $\mathrm{T}$ cells in the lesion (CD3; Figure 6B) expressed high levels of Arhgef1, compared with B cells (CD20; Figure 6B) or macrophages (C68; Figure 6B), and this was confirmed by quantification using Pearson's correlation coefficient analysis (Figure 6B).

\section{Discussion}

In summary, our data reveal a proinflammatory and pro-atherosclerotic role of the RhoA GEF Arhgef1 in leukocytes, through its action on the regulation of the conformational state of $\beta_{2}$ integrins. As a signaling molecule linking AT1 receptor stimulation to the control of integrin activity, Arhgef1 contributes to the role of Ang II in vascular inflammation and leukocyte recruitment by conferring a chemokine-like action to Ang II. We thus identify Arhgef1 as a critical component of the molecular mechanisms responsible for the proinflammatory effect of Ang II that may be responsible, at least in part, for the role of Ang II in atherosclerosis.
Activation of Arhgef1/RhoA signaling by AT1 receptor stimulation is subsequent to the phosphorylation of the Tyr738 of Arhgef1 by the tyrosine kinase Jak2 $(16,17)$. The Jak family of tyrosine kinases, classically described as main transducers of cytokine and chemokine receptor signaling $(22,23)$, has been recently shown to affect leukocyte adhesion (19). Jak kinases mediate chemokineinduced $\beta_{2}$ integrin triggering to a high-affinity state in a RhoAdependent manner. The RhoA GEF Vav1 has been suggested to be partially responsible for the Jak-dependent RhoA activation and integrin affinity modulation induced by CXCL12 (19). However, because of the multiplicity of Rho GEFs ( $~ 70)$, different upstream signals or receptors use different Rho GEFs to activate Rho proteins $(24,25)$. Accordingly, depending on the upstream signal, different RhoA GEFs might be used to similarly activate RhoA, thus providing specificity to various signaling pathways that converge toward RhoA. Here we identified the key role of the Jak2-dependent RhoA GEF Arhgef1 in the RhoA module of integrin activa- 
$\mathbf{A}$
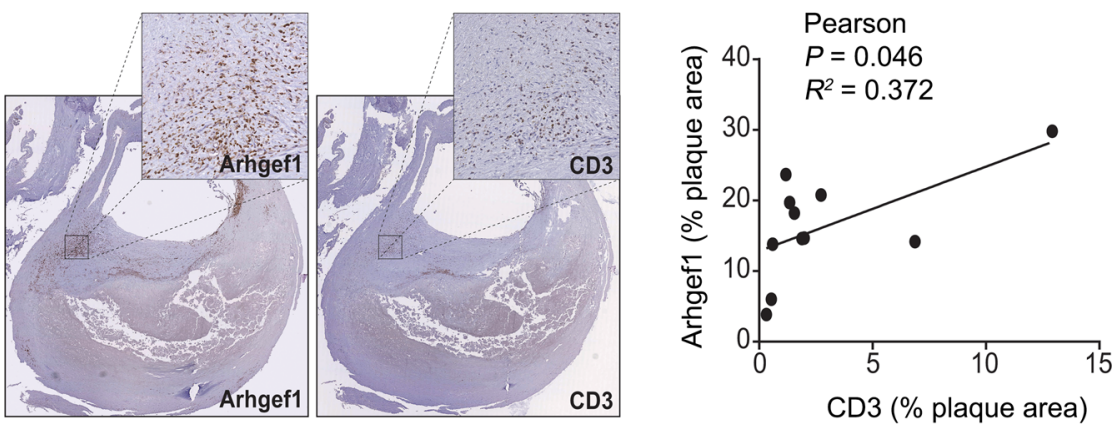

B
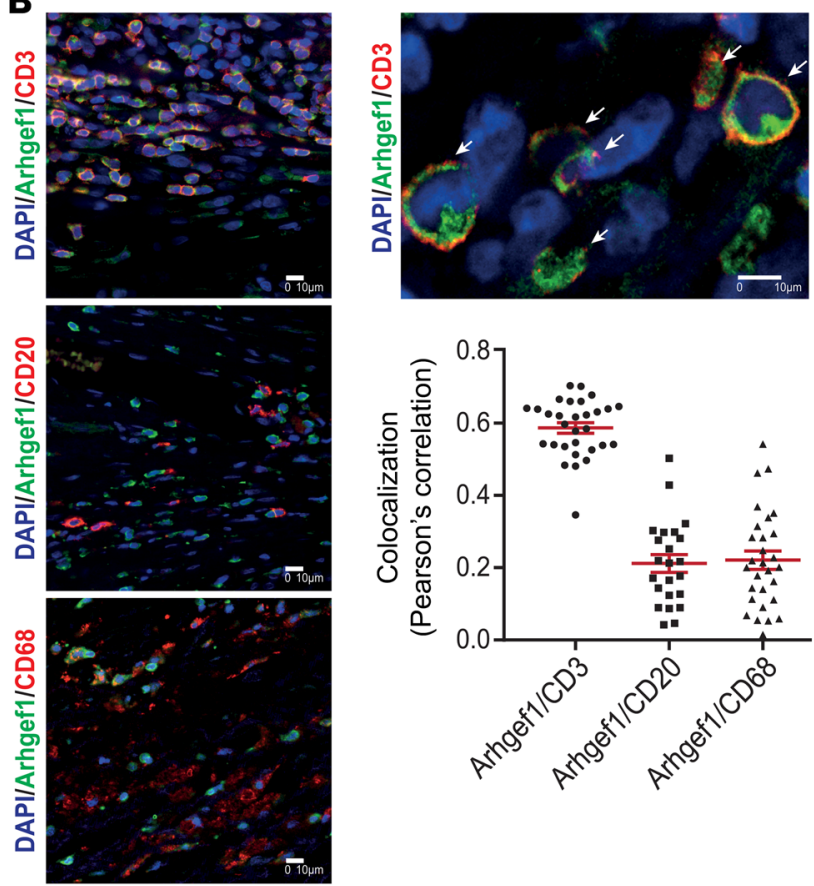

Figure 6. Immunostaining of Arhgef1 and immune cells in human carotid artery atherosclerotic lesion. (A) Immunohistochemical staining showing Arhgef1 and CD3 expression (in brown) in human atherosclerotic carotid artery sections and the correlation of their respective staining area relative to the whole lesion (original magnification, $\times 2.5$ ). The surrounded area in the complete section is displayed with higher magnification above $(\times 20)$. (B) Representative coimmunofluorescent staining of Arhgef1 with CD3 (top, T cells), CD20 (middle, B cells), and CD68 (bottom, macrophages), and corresponding plots of Pearson's correlation coefficients for the colocalization analysis. Colocalization of Arhgef1 and the T cell marker CD3 is illustrated in the higher-magnification image above the graph (arrows). Nuclei are stained with DAPI. Scale bars: $10 \mu \mathrm{m}$.

tion in response to Ang II and AT1 receptor stimulation. However, as Ang II stimulates secretion of cytokines and chemokines that themselves activate the Jak2 pathway, we cannot exclude that other soluble mediators such as CXCL12 contribute to Jak2-mediated Arhgef1 activation in addition to Ang II, thus perpetuating or potentiating the effect of Ang II on Arhgef $1 / \beta_{2}$ integrin signaling (26). In such cases, the decrease in circulating proinflammatory cytokines and chemokines observed in Arhgef1 $1^{-/-}$mice could also indirectly account for the protecting effect of Arhgef1 deletion against vascular inflammation and atherosclerosis.

Our results show that Arhgef1 deletion caused both a basal increase in $\beta_{2}$ integrin expression and a loss of its activation by Ang II. The control of the availability of integrins at the plasma membrane is key to their function, and internalization/recycling of inte- grins through the endosomal system plays an important role in the regulation of the plasma membrane pool of integrins (27). Integrin trafficking requires the spatial and temporal coordination of multiple molecules, including RhoA, which impacts on integrin trafficking by modulating actin cytoskeleton dynamics (28). While our data indicate that Arhgef1, downstream of AT1 receptor, is involved in Ang II-induced $\beta_{2}$ integrin activation, the observed increase in the pool of membrane $\beta_{2}$ integrins associated with Arhgef1 deletion is consistent with a role of Arhgefl/RhoA signaling in integrin trafficking.

Although the role of RhoA in vascular inflammation and atherosclerosis has not been directly addressed, evidence, mainly supported by the beneficial effect of Rho kinase inhibitors, suggested that RhoA/ Rho kinase signaling in vascular and hematopoietic cells participates in multiple steps of atherogenesis, including endothelial cell activation and dysfunction; leukocyte recruitment and cytokine and chemokine release; and oxidized LDL uptake in macrophage and foam cell formation (29). By showing a restricted role of Arhgef1 in leukocytes and in their recruitment to the endothelium, our data support the concept that RhoA GEFs discriminate upstream signals and that, depending on the cell type and the upstream activator, the RhoA GEF responsible for RhoA activation is different.

The high expression of Arhgefl in T cells in human carotid atherosclerotic lesions is in agreement with our results in mice and suggests that Arhgef1 might play a role in human atherosclerotic disease similar to that identified in mice. However, specific analysis in humans is necessary to validate these findings. It will be of particular interest to examine whether the content of inflammatory cells of atherosclerotic lesions is reduced in patients receiving statins compared with patients treated with other therapies, as statins inhibit leukocyte RhoA/Rho kinase signaling independently of cholesterol reduction in patients with atherosclerosis (30). A similar analysis in atherosclerotic lesions from patients treated with AT1 receptor antagonists or angiotensin-converting enzyme inhibitors would also be of great value.

We have previously described that Arhgef1 plays a major role in Ang II/AT1 receptor-induced RhoA activation in smooth muscle cells, vasoconstriction, and hypertension (17), and that Arhgef1/ RhoA signaling is turned on by RAAS activation in humans (16). The present findings showing the causal role of Arhgef1 in atherosclerosis further highlight the importance of Arhgef1 in cardiovascular disease and the potential interest of its targeting to treat or limit hypertensive and atherosclerotic cardiovascular disease. 


\section{Methods}

Animal studies. All mice used were backcrossed onto a C57BL/6N background at least 8-10 times. Arhgef1 ${ }^{\text {lox/lox }}$ C57BL/6 mice were generated as previously described (31). Arhgef1 $1^{\text {lox/lox }}$ were mated to CMV-Cre deleter mice [B6.C- $\mathrm{Tg}(\mathrm{CMV}$-Cre $) 1 \mathrm{Cgn} / \mathrm{J}$; The Jackson Laboratory] to obtain constitutive Arhgef1 ${ }^{-/}$mice. We generated $L D L R^{-/}$Arhgef1 ${ }^{-/}$mice by mating $L D L R^{-/-}$(The Jackson Laboratory) with Arhgef1 $1^{-/-}$mice. Mice had free access to food and water under a 12-hour light/12-hour dark cycle in a temperature-controlled environment. Mouse genotype was checked by PCR on tail sample DNA samples. When necessary, age-matched mice were divided into groups randomly. Losartan treatment $(16 \mathrm{mg} /$ $\mathrm{kg} / \mathrm{d}$ ) was administrated in the drinking water. For induction of atherosclerosis, 8-week-old mice were fed a semipurified cholate-free high-fat diet containing 40\% kcal lipid, 1.25\% cholesterol ad libitum (D12108c, Research Diets Inc.) for 12 weeks (32). Systolic blood pressure was measured by computerized tail cuff plethysmography in trained conscious mice (BP 2000, Visitech Systems). Mice were trained for 5 consecutive days to acclimate them to the apparatus. Computer-recorded measurements were then taken for 5 consecutive days following training. A minimum of 10 blood pressure readings per mouse per day were used to calculate the average daily blood pressure. The average blood pressure for each mouse was then calculated by averaging of the daily blood pressure of each mouse over the 5 consecutive days of readings.

Plasma samples were collected for lipid analysis after 6 hours of fasting before (week 0), at week 6, and at the end of the regimen (week 12). Plasma cholesterol levels were measured with RTU cholesterol kits (Biomerieux). Lipoproteins were isolated by fast protein liquid chromatography (FPLC). Two hundred microliters of plasma was injected into an MV-7 multi-injection loop, and separation was performed on 2 Superose 6 HR 10/30 columns in series with an elution flow rate of $0.35 \mathrm{ml} / \mathrm{min}$; $0.5 \mathrm{ml}$ was collected for each fraction, and the entire profile was completed within 105 minutes. The system was controlled by FPLC Director software (Amersham Pharmacia Biotech Inc.). Cholesterol levels were measured on FPLC fractions using RTU cholesterol kits (Biomerieux).

Intravital microscopy. All mice were used between 8 and 10 weeks of age. Mice were anesthetized with an i.p. injection of a mixture of xylazine (10 mg/kg; Bayer) and ketamine $(100 \mathrm{mg} / \mathrm{kg}$; Merial). A small midline abdominal incision was made, and a short segment of the small bowel was exteriorized, carefully avoiding stretching, and spread over a Plexiglas plate maintained at $37^{\circ} \mathrm{C}$. The exposed mesentery was superfused continuously with a warmed bicarbonate-buffered saline ( $\mathrm{pH}$ 7.4). Leukocytes were stained in vivo by an intracaudal injection of $1 \mathrm{mg} / \mathrm{ml}$ solution of fluorescent dye Rhodamine 6G (MilliporeSigma). A macroscope (Leica Z16 Plan APO 5.0 $/ 0.50$ LWD) was used to examine the mesenteric microcirculation. A high-speed video camera (ORCA-D2, Hamamatsu) mounted on the microscope projected the image onto a color monitor, and the images were recorded for offline analysis. The video images were processed and analyzed with Leica MM AF Imaging System software (MetaMorph). To measure leukocyte rolling and adhesion, 5 single unbranched mesenteric postcapillary venules (20-40 $\mu \mathrm{m}$ in diameter) were analyzed in each mouse according to methods previously described (33). The number of rolling leukocytes was determined by counting of leukocytes moving along the endothelial surface passing a line perpendicular to the vessel axis at a slower velocity than the stream of erythrocytes, and the leuko- cyte rolling flux was expressed as leukocytes per minute. Adherent leukocytes were defined as the total number of leukocytes firmly attached to the endothelium that remained stationary for at least 30 seconds, and were scored as the number of adherent cells within a 100- $\mu \mathrm{m}$ length of vessel. Analysis of leukocyte rolling and adhesion was made before (0) and 1, 4, and 8 hours after i.p. Ang II injection (0.1 pmol and 30 pmol; MilliporeSigma).

Western blot analysis. Cleaned mouse aortas were incubated on ice with lysis buffer supplemented with proteases and phosphatase inhibitors cocktails (MilliporeSigma) and sodium orthovanadate. Lysates were subjected to SDS-PAGE, transferred to nitrocellulose membranes, and incubated with specific antibodies (VCAM1 antibody, sc-1504, and ICAM1 antibody, sc-1511, Santa Cruz Biotechnology). Equal loading was checked by reprobing of the membrane with $\mathrm{mAb}$ to $\beta$-actin (A-5316, MilliporeSigma). Immune complexes were detected with appropriate secondary antibodies and enhanced chemiluminescence reagent (ECL Plus, GE Healthcare). Protein band intensities were quantified using ImageJ software (NIH).

Static adhesion assays on ICAM1. Immulon 1B Microtiter 96-well flat-bottom plates (Thermo Fisher Scientific) were coated overnight at $4^{\circ} \mathrm{C}$ with $2 \mu \mathrm{g} / \mathrm{ml}$ of ICAM1 (recombinant mouse ICAM1/CD54 Fc chimera, R\&D Systems). Nonspecific sites were then blocked with PBS containing 1\% BSA (MilliporeSigma) for 1 hour at room temperature and unbound protein removed by 4 washes with 3\% BSA in RPMI-1640 (Gibco). Peripheral blood mononuclear cells (PBMCs) were isolated by separation over Ficoll-Paque Plus (GE Healthcare) and resuspended in RPMI-1640 at a concentration of $5 \times 10^{4}$ cells per $100 \mu \mathrm{l}$. PBMCs were incubated with or without Ang II $\left(10^{-7} \mathrm{M}\right)$ for 4 hours at $37^{\circ} \mathrm{C}$, labeled with $1 \mu \mathrm{M} 2^{\prime}, 7^{\prime}$-bis-(2-carboxyethyl)-5-(and-6)carboxyfluorescein acetoxymethyl $\left(30\right.$ minutes at $37^{\circ} \mathrm{C}$; BCECF-AM, Molecular Probes), washed by centrifugation, then resuspended in RPMI-1640 with 10\% heat-inactivated FBS (MilliporeSigma). PBMCs were transferred into ICAM1-coated plates at $5 \times 10^{4}$ cells per well and incubated for 10 minutes at $37^{\circ} \mathrm{C}$. The plates were washed 4 times with $0.9 \% \mathrm{NaCl}$ and fluorescence emitted from attached cells was measured with VICTOR X3 Multilabel Plate Reader (PerkinElmer).

In vitro flow assays. HUVECs (PromoCell) were cultured in Endothelial Cell basal medium MV supplemented with 5\% FCS (PromoCell). HUVECs were seeded at $2 \times 10^{5}$ cells into Ibidi $\mu$-slide I 0.4 Luer (Ibidi) coated with ibiTreat, and cultivated under flow conditions with a gradient of shear stress ( $3.42 \mathrm{dyn} / \mathrm{cm}^{2}$ for 30 minutes, $5 \mathrm{dyn} / \mathrm{cm}^{2}$ for 30 minutes, $10 \mathrm{dyn} / \mathrm{cm}^{2}$ for 30 minutes, $15 \mathrm{dyn} / \mathrm{cm}^{2}$ for 1 hour, $20 \mathrm{dyn} /$ $\mathrm{cm}^{2}$ for 1 hour, $25 \mathrm{dyn} / \mathrm{cm}^{2}$ for 48 hours). HUVECs were activated with TNF- $\alpha(5 \mathrm{ng} / \mathrm{ml}) 4$ hours before the PBMC adhesion assay. PBMCs, prepared as described above, were then stained by $1 \mathrm{mg} / \mathrm{ml}$ solution of fluorescent dye Rhodamine 6G (MilliporeSigma) and perfused at 0.79 $\mathrm{dyn} / \mathrm{cm}^{2}$ (perfusion set). Ibidi $\mu$-slides were placed on a macroscope (Leica Z16 Plan APO 5.0×/0.50 LWD), and videos were recorded by a high-speed video camera (ORCA-D2, Hamamatsu) for offline analysis. The video images were processed and analyzed with Leica MM AF Imaging System software (MetaMorph).

Flow cytometry. Blood samples $(100 \mu \mathrm{l})$ were obtained by EDTA-anticoagulated retro-orbital sinus collection in isofluraneanesthetized mice. Red cells were lysed using BD Pharm Lyse lysing buffer (BD Biosciences). Leukocytes were stained with PerCP/Cy5.5CD11a/CD18, Brilliant Violet 421-CD11b, and Alexa Fluor 647-CD18 (all from BioLegend) at $4^{\circ} \mathrm{C}$ for 30 minutes in the dark. The appropriate 
isotype controls were used for compensation settings to estimate nonspecific staining of primary antibodies (PerCP/Cy5.5 rat IgG1, 421 rat IgG2b, Alexa Fluor 647 rat IgG2a, all from BioLegend). To study the integrin $\beta_{2}$-active form, leukocytes were stained with FITC-conjugated mouse mAb 24, which recognizes the high-affinity conformation (Abcam). Flow cytometry analysis was performed with a FACS LSR II flow cytometer equipped with FACSDiVA 6.3.1 software (BD Biosciences). A minimum of 50,000 events was acquired from each sample. Results were expressed as percent positive cells of total leukocytes.

For mouse aorta analysis, Ldlr/-Arhgef1 ${ }^{\text {lox/lox }}$ and Ldlr/-Arhgef1 $1^{-1-}$ aortas were harvested after 12 weeks under high-fat diet. Aortas were cut in small pieces and then digested with an enzymatic cocktail of collagenase I (450 U/ml), hyaluronidase (60 U/ml), DNase I (60 U/ml), and collagenase XI (120 U/ml) (MilliporeSigma) in PBS containing 20 mM HEPES, for 90 minutes at $37^{\circ} \mathrm{C}$ with stirring. Aorta cell suspensions were then filtered through a $70-\mu \mathrm{m}$ cell strainer (BD Biosciences) and washed with PBS, FBS 5\%, EDTA 1\%. Cells were then counted and stained with the following antibodies: PEcy7-CD3, PE-CD45, APC-CD4, FITC-F4/80, and APC-H7-CD19 (BD Biosciences), in the presence of a viability dye, LIVE/DEAD Aqua dye (Life Technologies). Cell suspensions were analyzed using a FACS Fortessa X20 (BD Biosciences), and data were analyzed using FlowJo (Tree Star).

$R N A$ isolation from mouse aortas and quantitative reverse transcriptase PCR. Total RNA from mouse thoracic aortas was extracted using RNA STAT60 (AMSBIO) according to the manufacturer's instructions. Genomic DNA was digested using a DNA-free kit (Ambion). Total RNA was reverse transcribed using SuperScript IV VILO master mix (Invitrogen), and PCR was performed using MESA GREEN MasterMix Plus, ROX (Eurogentec), with the 7900HT Fast Real-Time PCR System (Applied Biosystems). Primer sequences used were as follows: Adgre1, forward, CTTTGGCTATGGCTTCCAGTC; Adgre1, reverse, GCAAGGAGGACAGAGTTTATCGTG; Ill b, forward, TGGGCCTCAAAGGAAAGAAT; $I l 1 b$, reverse, CAGGCTTGTGCTCTGCTTGT; Tnfa, forward, CCAGACCCTCACACTCAGATC; Tnfa, reverse, CACTTGGTGGTTTGCTACGAC. Levels of mRNA expression were normalized to the ribosomal protein $36 B 4$ mRNA expression (36B4, forward, AGATGCAGCAGATCCGCAT; 36B4, reverse, GTTCTTGCCCATCAGCACC).

Analysis of atherosclerotic lesions. After 12 weeks of diet, the mice were anesthetized with i.p. injection of a mixture of xylazine/ketamine with heparin, and blood was collected by left ventricular puncture into a syringe containing EDTA for plasma recuperation. Hearts and aortas were flushed by intraventricular perfusion of PBS $(5 \mathrm{ml})$ followed by $4 \%$ paraformaldehyde (PFA; $2 \mathrm{ml}$ ). The entire aortas attached to the hearts were dissected, placed overnight in $4 \% \mathrm{PFA}$ at $4^{\circ} \mathrm{C}$, and then separated between the aortic valve and arch. Aortas were cleaned from adventitial fat for en face Oil Red O staining. Oil Red O staining $(0.2 \%, 1$ hour at room temperature; MilliporeSigma) was preceded and followed by 2 washes with 78\% methanol. After staining, the remaining adventitial fat was easily detected and removed. The aortas were opened longitudinally, and the images were collected by Leica M80 microscope equipped with IC80 HD digital camera and LAS software version 4.3 (Leica). Hearts were incubated overnight in phosphate-buffered $20 \%$ sucrose solution at $4^{\circ} \mathrm{C}$, then embedded in Tissue-Tek OCT compound (Sakura Finetek) and frozen in isopentan/ liquid nitrogen. Serial 8- $\mu \mathrm{m}$-thick cross-cryosections of the aortic root were cut from the point of appearance of the 3 aortic valve leaflets. For lesion area analysis, sections were stained with Oil Red O (0.2\%), and images were captured using a Nikon Eclipse E600 light microscope equipped with a Nikon DS-Ri1 camera. The lesion area in each mouse was then quantified by averaging of measurements from 6 sections 50 $\mu \mathrm{m}$ apart using ImageJ software. The intimal lesion area was expressed as square millimeters.

OxLDL uptake assay and foam cell formation. Peritoneal macrophages were collected 3 days after i.p. injection of thioglycolate. Macrophages were cultured in 10\% FCS-RPMI for 24 hours and then used in various experiments. For oxLDL uptake assay, human oxLDL (AbD Serotec) was incubated with $1 \mu \mathrm{Ci} / \mathrm{ml}{ }^{3} \mathrm{H}$-cholesterol at a final concentration of $50 \mu \mathrm{g} / \mathrm{ml}$ in $0.2 \%$ BSA-RPMI for 30 minutes at $37^{\circ} \mathrm{C}$. Macrophages were treated with the tritiated oxLDL for 3 hours, and radioactivity was counted. For BODIPY staining, cells were washed twice in PBS, fixed in 4\% PFA for 5 minutes, and then stained with BODIPY 493/503 (Life Technologies) for 30 minutes. For foam cell formation, macrophages were incubated 48 hours in 10\% FCS-RPMI supplemented with $100 \mu \mathrm{g} / \mathrm{ml}$ oxLDL (AbD Serotec).

Immunohistological and immunofluorescent analysis of atherosclerotic lesion. Immunofluorescence labeling of mouse atherosclerotic lesions was performed on frozen aortic mouse sinus sections after permeabilization (PBS 0.3\% Triton-X100; 10 minutes). Sections were blocked with $3 \%$ BSA for 1.5 hours and incubated with anti-CD3-PE antibody (1:100; 12-0030-83, eBioscience) and anti-Arhgef1/p115/ Lsc antibody (H-165) (1:200; sc-20804, Santa Cruz Biotechnology) overnight at $4{ }^{\circ} \mathrm{C}$ followed by incubation with Alexa Fluor 568-conjugated goat anti-rabbit $(1: 2,000)$, or with or anti-CD68 (1:100; MCA1957GA, Bio-Rad) and anti-Arhgef1/p115/Lsc antibody (1:200) overnight at $4^{\circ} \mathrm{C}$, followed by incubation with Alexa Fluor 488-conjugated goat anti-rat $(1: 1,000)$ and Alexa Fluor 568-conjugated goat anti-rabbit (1:2,000). Negative controls were performed without respective primary antibodies. Images of the sections were captured with a fluorescence microscope (Eclipse E-600, Nikon Instruments Inc.) fitted with a DS-Ri1 camera and NIS-Elements BR 4.1 software (Nikon Instruments Inc.). At least 4 sections per mouse were examined for each immunostaining. CD68-positive areas were quantified using ImageJ software.

Analysis in humans was performed on carotid artery lesions $(n=11)$. Carotid plaques were removed by endarterectomy at the bifurcation from within the lumen as a single specimen. The atherosclerotic plaques and spleen sample were fixed in 10\% formalin for 48 hours, decalcified in 4.13\% EDTA-0.2\% PFA, pH 7.4, over 4 days in KOS sw10 (Milestone), and embedded in paraffin. Labeling of $4-\mu \mathrm{m}$-thick serial sections was then performed following heat-induced epitope retrieval in EDTA, pH 9. Image acquisition of immunohistochemical staining of CD3 (F2.2.38, Dako) and Arhgef1 (H-165, Santa Cruz Biotechnology) was done with a whole-slide scanner NanoZoomer (Hamamat$\mathrm{su}$. Quantification of respective staining area relative to the whole lesion was performed with Image-Pro Plus (Media Cybernetics), and statistical correlation was assessed with Prism (GraphPad) software. For immunofluorescence analysis, sections were costained for CD3 (F2.2.38, Dako), CD20 (L26, Abcam), or CD68 (PG-M1, Dako) and Arhgef1 (H-165, Santa Cruz Biotechnology), and nuclei were labeled with DAPI (Invitrogen/Thermo Fisher Scientific). Biotin-, Alexa Fluor 488-, and Alexa Fluor 568-conjugated secondary antibodies were purchased from Dako and Life Technologies. Negative controls were performed without respective primary antibodies. Image acquisition was done by a NanoZoomer slide scanner (Hamamatsu), and by a con- 
focal microscope (Nikon A1 RSi), using an original magnification $\times 60$ 1.4 oil $p$-aplo lens. Quantification of colocalization was performed with Volocity (PerkinElmer) on at least twenty-four 20x-equivalent fields of views for each costaining.

BM transplantation. Eight-week-old mice received nonlethal totalbody irradiation (Faxitron CP-160 x-ray, 9 Gy) 24 hours before transplant. BM cells were harvested from long leg bones of donors by gentle saline flush, washed once, and retro-orbitally injected as unfractionated viable cell mixture in normal saline $\left(10 \times 10^{6}\right.$ per mouse $)$ in mice anesthetized with isoflurane $(4 \%$, then $2 \% ; 11 / \mathrm{min})$. The mice were used for experiments 4 weeks after transplantation. The successful BM engraftment was monitored by Arhgef1 PCR on DNA from blood leukocytes and tail samples from the irradiated/reconstituted mice.

Statistics. Data are expressed as the mean \pm SEM of sample size $n$. All values use biological replicates and are indicated by group size $n$ in figure legends or within graphs. For in vivo or ex vivo data, each $n$ value corresponds to a single mouse. For in vitro data, each $n$ value corresponds to an independent experiment. If technical replicates were performed, then their mean was considered as an $n=1$. No statistical method was used to predetermine sample size that was based on preliminary data. No samples were excluded from the analysis. Investigators were blinded for some measurements made in mice (i.e., blood cell count, histology, lesion area, lipid measurement). Comparisons between 2 groups were performed by unpaired, 2-tailed nonparametric Mann-Whitney $U$ test, and 1-way ANOVA with relevant post hoc tests was used for multiplegroup comparisons using GraphPad Prism 6.0 software (GraphPad Software). $P$ less than 0.05 was considered statistically significant.

Study approval. All animal care and use procedures of the present study were performed in accordance with the European Community Standards on the Care and Use of Laboratory Animals and were approved by our institutional ethics committee (Comité d'éthique en expérimentation animale des Pays de la Loire no. 6) (project autho- rization 00910.05). For the analysis of human carotid samples, all patients participating in the study gave written informed consent. The clinical research protocol was approved by our institutional medical ethics committee (Nantes University Hospital Ethics Committee).

\section{Author contributions}

MLC contributed to study design, and performed all experiments and data analysis with GC, SHC, JA, NV, MR, CBM, and AT. XP performed experiments and analyzed data on lipid metabolism and participated in experiments in atherosclerosis models. TQ and YG collected and performed experiments on human samples. LC, MD, and JD performed flow cytometry experiments. RMT generated Arhgef1 ${ }^{l o x} / l o x$ mice. GL planned and directed the study, and wrote the manuscript.

\section{Acknowledgments}

We thank Therassay (IBISA/Biogenouest, Nantes) and CytoCell (Biogenouest, Nantes) platforms for the functional explorations in mice and flow cytometry, respectively. We also value the support provided by the animal facility unit (Unité Thérapeutique Expériementale [UTE]) of the University of Nantes and the assistance of the cellular and tissue imaging core facility of Nantes University (MicroPICell) for immunofluorescence imaging. This work was supported by the Institut National de la Santé et de la Recherche Médicale (INSERM), the Fondation de France ( $\mathrm{N}^{\circ}$ Engt: 201300038590), and the Institut de Recherches Servier. MLC was supported by grants from Laboratorio di Genomica e Proteomica Funzionale, Universita di Bari, Bari, Italia.

Address correspondence to: Gervaise Loirand, INSERM 1087, IRSUN, 8 Quai Moncousu, BP 70721, 44007 Nantes cedex 1, France. Phone: 33.2.28.08.01.16; Email: gervaise.loirand@univ-nantes.fr.
1. de Gasparo M, Catt KJ, Inagami T, Wright JW, Unger T. International union of pharmacology. XXIII. The angiotensin II receptors. Pharmacol Rev. 2000;52(3):415-472.

2. Touyz RM, Berry C. Recent advances in angiotensin II signaling. Braz J Med Biol Res. 2002;35(9):1001-1015.

3. Ferrario CM, Strawn WB. Role of the renin-angiotensin-aldosterone system and proinflammatory mediators in cardiovascular disease. Am J Cardiol. 2006;98(1):121-128.

4. Cassis LA, Rateri DL, Lu H, Daugherty A. Bone marrow transplantation reveals that recipient AT1a receptors are required to initiate angiotensin II-induced atherosclerosis and aneurysms. Arterioscler Thromb Vasc Biol. 2007;27(2):380-386.

5. Daugherty A, Manning MW, Cassis LA. Angiotensin II promotes atherosclerotic lesions and aneurysms in apolipoprotein E-deficient mice. J Clin Invest. 2000;105(11):1605-1612.

6. Daugherty A, Rateri DL, Lu H, Inagami T, Cassis LA. Hypercholesterolemia stimulates angiotensin peptide synthesis and contributes to atherosclerosis through the AT1A receptor. Circulation. 2004;110(25):3849-3857.

7. Fukuda D, Sata M, Ishizaka N, Nagai R. Critical role of bone marrow angiotensin II type 1 receptor in the pathogenesis of atherosclerosis in apo- lipoprotein E deficient mice. Arterioscler Thromb Vasc Biol. 2008;28(1):90-96.

8. Johnstone MT, et al. Angiotensin receptor blockade with candesartan attenuates atherosclerosis, plaque disruption, and macrophage accumulation within the plaque in a rabbit model. Circulation. 2004;110(14):2060-2065.

9. Lu H, Daugherty A. Atherosclerosis: cell biology and lipoproteins. Curr Opin Lipidol. 2012;23(3):263-264.

10. Strawn WB, Chappell MC, Dean RH, Kivlighn S, Ferrario CM. Inhibition of early atherogenesis by losartan in monkeys with diet-induced hypercholesterolemia. Circulation. 2000;101(13):1586-1593.

11. Wassmann S, Czech T, van Eickels M, Fleming I, Böhm M, Nickenig G. Inhibition of diet-induced atherosclerosis and endothelial dysfunction in apolipoprotein E/angiotensin II type 1A receptor double-knockout mice. Circulation. 2004;110(19):3062-3067.

12. Mansur SJ, Hage FG, Oparil S. Have the reninangiotensin-aldosterone system perturbations in cardiovascular disease been exhausted? Curr Cardiol Rep. 2010;12(6):450-463.

13. Pacurari M, Kafoury R, Tchounwou PB, Ndebele $\mathrm{K}$. The renin-angiotensin-aldosterone system in vascular inflammation and remodeling. Int J Inflam. 2014;2014:689360.
14. Zhou Q, Gensch C, Liao JK. Rho-associated coiled-coil-forming kinases (ROCKs): potential targets for the treatment of atherosclerosis and vascular disease. Trends Pharmacol Sci. 2011;32(3):167-173.

15. Montecucco F, Mach F. Update on statin-mediated anti-inflammatory activities in atherosclerosis. Semin Immunopathol. 2009;31(1):127-142.

16. Carbone ML, et al. Angiotensin II activates the RhoA exchange factor Arhgef1 in humans. Hypertension. 2015;65(6):1273-1278.

17. Guilluy C, et al. The Rho exchange factor Arhgef1 mediates the effects of angiotensin II on vascular tone and blood pressure. Nat Med. 2010;16(2):183-190.

18. Hogg N, Patzak I, Willenbrock F. The insider's guide to leukocyte integrin signalling and function. Nat Rev Immunol. 2011;11(6):416-426.

19. Montresor A, Bolomini-Vittori M, Toffali L, Rossi B, Constantin G, Laudanna C. JAK tyrosine kinases promote hierarchical activation of Rho and Rap modules of integrin activation. J Cell Biol. 2013;203(6):1003-1019.

20. Montresor A, Toffali L, Constantin G, Laudanna C. Chemokines and the signaling modules regulating integrin affinity. Front Immunol. 2012;3:127.

21. Merched A, Tollefson K, Chan L. $\beta 2$ Integrins mod- 


\section{RESEARCH ARTICLE}

ulate the initiation and progression of atherosclerosis in low-density lipoprotein receptor knockout mice. Cardiovasc Res. 2010;85(4):853-863.

22. Soriano SF, et al. Chemokines integrate JAK/ STAT and G-protein pathways during chemotaxis and calcium flux responses. Eur J Immunol. 2003;33(5):1328-1333.

23. Stein JV, et al. CCR7-mediated physiological lymphocyte homing involves activation of a tyrosine kinase pathway. Blood. 2003;101(1):38-44.

24. García-Mata R, Burridge K. Catching a GEF by its tail. Trends Cell Biol. 2007;17(1):36-43.

25. Rossman KL, Der CJ, Sondek J. GEF means go: turning on RHO GTPases with guanine nucleotide-exchange factors. Nat Rev Mol Cell Biol. 2005;6(2):167-180.
26. Toffali L, Montresor A, Mirenda M, Scita G, Laudanna C. SOS1, ARHGEF1, and DOCK2 rhoGEFs mediate JAK-dependent LFA-1 activation by chemokines. J Immunol. 2017;198(2):708-717.

27. Caswell PT, Norman JC. Integrin trafficking and the control of cell migration. Traffic. 2006;7(1):14-21.

28. Ridley AJ. Rho GTPases and actin dynamics in membrane protrusions and vesicle trafficking. Trends Cell Biol. 2006;16(10):522-529.

29. Loirand G, Sauzeau V, Pacaud P. Small G proteins in the cardiovascular system: physiological and pathological aspects. Physiol Rev. 2013;93(4):1659-1720.

30. Nohria A, et al. Statins inhibit Rho kinase activity in patients with atherosclerosis. Atherosclerosis.
2009;205(2):517-521.

31. Rubtsov A, Strauch P, Digiacomo A, Hu J, Pelanda $\mathrm{R}$, Torres RM. Lsc regulates marginal-zone $\mathrm{B}$ cell migration and adhesion and is required for the IgM T-dependent antibody response. Immunity. 2005;23(5):527-538.

32. Lichtman AH, Clinton SK, Iiyama K, Connelly PW, Libby P, Cybulsky MI. Hyperlipidemia and atherosclerotic lesion development in LDL receptor-deficient mice fed defined semipurified diets with and without cholate. Arterioscler Thromb Vasc Biol. 1999;19(8):1938-1944.

33. Abu Nabah YN, et al. CXCR2 blockade impairs angiotensin II-induced CC chemokine synthesis and mononuclear leukocyte infiltration. Arterioscler Thromb Vasc Biol. 2007;27(11):2370-2376. 